Check for updates

Cite this: RSC Adv., 2021, 11, 9586

Received 16th December 2020 Accepted 26th February 2021

DOI: $10.1039 / d 0 r a 10565 a$

rsc.li/rsc-advances

\section{Effect of silylating agents on the superhydrophobic and self-cleaning properties of siloxane/ polydimethylsiloxane nanocomposite coatings on cellulosic fabric filters for oil-water separation $\uparrow$}

\begin{abstract}
Ubong Eduok, (D)*a Omar Faye, ${ }^{a}$ Jerzy Szpunar ${ }^{a}$ and Mazen Khaled ${ }^{b}$
A new facile approach for preparing two nonfluorinated hybrid organic-inorganic siloxane/ polydimethylsiloxane nanocomposite coatings for cotton fabrics is presented using two distinct silylating agents. The first coated fabric was prepared predominantly via trimethylsilyl modification using hexamethyldisilazane (HMDS) while higher amounts of trimethoxy(octadecyl)silane (TMOS) further enhanced the superhydrophobicity of the second coating matrix. Unlike HMDS with substituted silyl $\left(\mathrm{Me}_{3} \mathrm{Si}\right)$ groups, TMOS consists of hydrolysable trimethoxy silyl $\left((\mathrm{MeO})_{3} \mathrm{Si}\right)$ chemical groups that allowed for the formation of nanosilica with $\mathrm{Si}-\mathrm{O}-\mathrm{Si}$ linkages needed to foster stable coatings. After characterization and testing, these coated fabrics demonstrated varying responses to harsh solvents and thermal conditions. Both sets of coated fabrics exhibited unique capacities for self-cleaning and oilwater separation as superhydrophobic filters due to (a) their low surface energy silylated hybrid polysiloxane chemical groups, (b) their highly reduced surface wettability and (c) nanopatterned surface morphologies. In this study, coated superhydrophobic cotton fabrics revealed a higher static aqueous contact angle $\left(\theta_{\mathrm{W}}^{\circ}\right)$ of more than $150^{\circ}$ and sliding hysteresis angle $\left(\theta_{\mathrm{SH}}^{\circ}\right)$ of less than $5^{\circ}$. Coated fabrics with $30 \mathrm{mg}$ TMOS/10 mg HMDS (CMF3) and $30 \mathrm{mg} \mathrm{HMDS} / 10 \mathrm{mg}$ TMOS (CTF3) exhibited optimal superhydrophobicity. Both fabrics also retained percentage separation efficiencies over $90 \%$ for both chloroform-water and toluene-water mixtures. However, CTF3 displayed $\theta_{\mathrm{w}}^{\circ}<150^{\circ}$ with a recorded separation efficiency less than $90^{\circ}$ after five filtration cycles.
\end{abstract}

\section{Introduction}

Recent trends in human activities have led to frequent oil spills and waste disposals, and these have severely impacted on marine lives. ${ }^{1}$ Discharges from industrial wastewaters generate oil-based environmental eluents (e.g., toluene) and watersoluble pollutants (e.g., dyes) whose dispersion within the food chain are also detrimental to human health. ${ }^{2}$ In cases of oil spills, the formation of organic films isolates the seawater from rapid oxygen exchange with the surrounding atmosphere while starving marine lives of the needed molecular oxygen sustenance. The challenges associated with effluent oil treatments using physical and chemical techniques as well as the complex nature of these effluents necessitate the design and development of functionalized filter materials capable of efficient and

${ }^{a}$ Department of Mechanical Engineering, College of Engineering, University of Saskatchewan, 57 Campus Drive, Saskatoon, S7N 5A9, Saskatchewan, Canada. E-mail: ubong.eduok@usask.ca; Fax: +1 306966 5427; Tel: +1 3069667752

${ }^{b}$ Department of Chemistry and Earth Sciences, College of Arts and Sciences, Qatar University, P.O. Box 2713, Doha, Qatar

$\dagger$ Electronic supplementary information (ESI) available. See DOI: 10.1039/d0ra10565a sustainable oil/water separation to remediate severe pollution incidents. The use of superhydrophobic filters has attracted special attention due to their unique surface properties, including low surface energies and self-cleaning capacity toward high separation efficiency between oil and water. Unlike most chemical treatment techniques, superhydrophobic filters utilized in physical separation techniques are environmentally friendly materials that are effective for liquid-liquid phase separations without the need for the use of specialized equipment. These materials reserve their unique recovery efficiencies for oil remnants and high oil-water separation capacity due to their nanostructured superhydrophobic surfaces with high hydrostatic aqueous contact $\left(\theta_{\mathrm{w}}^{\circ}\right)$ and low sliding hysteresis $\left(\theta_{\mathrm{SH}}^{\circ}\right)$ angles. Modern superhydrophobic filters are fabricated to achieve surfaces with exceptionally low surface energies capable of mimicking the self-cleaning lotus effect in Nelumbo nucifera (lotus) leaves, with minimized water and even oil droplet adhesions. $^{3}$ Like surfaces of the lotus leaf, these superhydrophobic filters are designed with non-wetting micro/ nanohierarchical papilla-leavened structures with superhydrophobic surfaces. ${ }^{4}$ These superhydrophobic filter surfaces are fabricated from various techniques involving physical 
changes, chemical depositions, spray, vapor and even laser surface modifications. ${ }^{5-8}$ Functional superhydrophobic filter surfaces fabricated from these techniques are also designed to possess defined textures with chemical properties capable of inducing extremely high-water repellency to which cotton fabrics are a classical example. Cotton fabrics are one of the most low-cost and efficient superhydrophobic filters since they consist of hydrophilic hydroxyl-bound cellulosic backbones capable of being chemically altered to introduce new functional groups that offer enhanced surface properties. ${ }^{9}$ They are also flexible and with enhanced tunable mechanical strengths.

There are a number of recent research reports and review articles featuring oil/water separation and self-cleaning properties of cellulosic superhydrophobic fabric materials. ${ }^{10-17}$ Guo et $a l^{14}$ investigated the efficiency of a superhydrophobic bilayer coating deposited on cotton fabric via spraying technique. The coating layers consisted of $\mathrm{TiO}_{2} / \mathrm{PDMS}$ and alkylammonium functional silsesquioxane/phytic acid hybrid composites. Results reported by authors revealed a coating system with significant capacity for self-cleaning and water-oil separation due to its superhydrophobic surface. The coating system with the best performance was abrasion resistant and also exhibited strong separation efficiency for strong acid- or alkali-oil mixtures. This last function stood at $\theta_{\mathrm{w}}^{\circ}>139^{\circ}$ after 50 abrasion cycles due to PDMS modification of the adhering $\mathrm{TiO}_{2}$ nanoparticles. Jannatun et al. ${ }^{5}$ have also reported the use of a PDMS-based coating system that utilizes boric acid $\left(\mathrm{H}_{3} \mathrm{BO}_{3}\right)$ as a cross-linker between silica nanoparticles and poly(vinyl alcohol). It was applied on the cotton fabric via dip coating technique. The presence of $\mathrm{SiO}_{2}$ nanoparticles within the coating also contributed to its oil-water separation and self-healing abilities. Variants of this coated fabrics revealed durability against mechanical abrasion (e.g., tape-peeling) and chemical stresses (e.g., corrosion solvents) with sufficiently unaltered values of $\theta_{\mathrm{w}}^{\circ}$ after several cycles. A magnitude of separation efficiency up to $90 \%$ was recorded during oil-water mixture separation between 5 to 45 separation cycles. In another study, Talebizadehsardari et al. ${ }^{13}$ investigated the efficiency of reusable oil/ water separation when using a superhydrophobic nanocomposite coated cotton fabric fabricated from PDMS and silica nanoparticles. Here, authors also probed the effect of curing agents on the oil/ water separation efficiencies of coated fabrics. They observed that curing agents contributed to the superhydrophobicity of each coating variants as more silica nanoparticles were adsorbed on the fibers of the fabric. Authors reported 97.6 and $95.1 \%$ separation efficiencies after 5 and 10 washing cycles, respectively.

One common highlight among these reported examples is the introduction of nanoparticles to promote superhydrophobicity. However, there is a pressing concern regarding failed coating adhesion when their particle sizes and concentrations are not sufficiently regulated. In the present study, steps are taken to further to achieve superhydrophobicity by addressing this inherent surface problem by modifying coated polymer chains with silylating agents. As a contribution to the use of reinforced coated superhydrophobic fabrics, the present study features a facile one-pot novel approach to synthesizing new siloxane/ PDMS nanocomposite coatings involving surface modification with two distinct silylating agents to achieve enhanced superhydrophobicity when deposited on cotton fabric. The two silylating agents are hexamethyldisilazane (HMDS) and trimethoxy(octadecyl)silane (TMOS). Unlike HMDS with substituted silyl $\left(\mathrm{Me}_{3} \mathrm{Si}\right)$ groups, TMOS consists of hydrolysable trimethoxy silyl $\left((\mathrm{MeO})_{3} \mathrm{Si}\right)$ chemical groups that allow for the formation of nanosilica with $\mathrm{Si}-\mathrm{O}-\mathrm{Si}$ linkages needed to foster stable coatings. Why use silylating agents? These precursors were introduced as silicon-based components capable of improving the surface hydrophobicity of the bulk coating via their substituted silyl group $\left(\mathrm{R}_{3} \mathrm{Si}\right)$ chemical groups. Here, the desired substituted silyl groups are introduced within molecular chains of the synthesized nanocomposite polymer in order to impart physical and chemical modifications to their surfaces. The major research highlight features the investigation of the effects of both silylating agents toward enhanced surface non-wetting, self-cleaning and oil-water separations. The surfaces of coated fabrics in the present study possess functional groups chemically grafted on to the cotton fabrics to offer low wettability and nanoporous and nanopatterned surface roughness.

\section{Experimental procedures}

\subsection{Reagents and chemicals}

The coating precursors in the present study were ethoxy terminated polydimethylsiloxane (EtPDMS, 5-10 cst, >95\%) and 3-(2,3epoxypropoxypropyl)methyldimethoxysilane (EPMM, 95-100\%), purchased from GELEST. Trimethoxy(octadecyl)silane (TMOS, $>95 \%$ ), hexamethyldisilazane (HMDS, $\geq 99 \%$ ) and chlorotrimethylsilane (TMCS, $\geq 97 \%$ ) were purchased from Sigma Aldrich and GELEST, alongside isopropyl alcohol (IPA, HPLC grade), acetone $(\geq 99.5 \%)$ and anhydrous ammonia $(\geq 99.98 \%)$. Sudan red G (analytical standard) and methyl orange (ACS reagent, $85 \%$ dye content) from Sigma Aldrich were utilized as dyes in this study. All the chemicals in the present study were used as purchased without further purification as they were analytical grade regents.

\subsection{Preparing silylated superhydrophobic silica nanocomposites}

Cotton fabrics were modified with coatings synthesized in situ a reaction medium with two different silylating agents, introduced to provide the needed surface hydrophobicity. The first step in preparing the PDMS coating involved refluxing a suspension of trimethoxy(octadecyl)silane (TMOS) and 3-(2,3epoxypropoxypropyl)methyldimethoxysilane (EPMM, $10 \mathrm{mg}$ ), in equimolar concentrations with ethoxy terminated polydimethylsiloxane $(10 \mathrm{mg})$ in $3 \mathrm{~mL}$ water and $2 \mathrm{~mL}$ IPA at $50-$ $55{ }^{\circ} \mathrm{C}$. The $\mathrm{pH}$ of the reaction medium was adjusted to $8 \mathrm{using}$ ammonia solution. After allowing hydrolysis-condensation reactions on respective alkoxide-bearing reactants for $8 \mathrm{~h}$, we proceeded with modification of the reaction medium with hexamethyldisilazane (HMDS) in the presence of chlorotrimethylsilane TMCS $(2: 0.5)$ at $40{ }^{\circ} \mathrm{C}$ for $3 \mathrm{~h}$ and stirred at $500 \mathrm{rpm}$. By this time, the resultant reaction medium turned viscous. Varying concentrations of HMDS and TMOS (10-40 mg) in the reaction media represent in coating variants in the 
Table 1 Coating labels and their description as utilized in the present study

\begin{tabular}{lll}
\hline $\begin{array}{l}\text { Coating } \\
\text { matrix }\end{array}$ & Variant labels/notations & Coating description \\
\hline CTF1-4 & CTF1 & $10 \mathrm{mg} \mathrm{HMDS} / 10 \mathrm{mg}$ TMOS \\
& CTF2 & $20 \mathrm{mg} \mathrm{HMDS} / 10 \mathrm{mg}$ TMOS \\
& CTF3 & $30 \mathrm{mg} \mathrm{HMDS} / 10 \mathrm{mg}$ TMOS \\
& CTF4 & $40 \mathrm{mg}$ HMDS $/ 10 \mathrm{mg}$ TMOS \\
CMF1-4 & CMF1 ${ }^{a}$ & $10 \mathrm{mg} \mathrm{TMOS} / 10 \mathrm{mg}$ HMDS \\
& CMF2 & $20 \mathrm{mg}$ TMOS $/ 10 \mathrm{mg}$ HMDS \\
& CMF3 & $30 \mathrm{mg} \mathrm{TMOS} / 10 \mathrm{mg}$ HMDS \\
& CMF4 & $40 \mathrm{mg}$ TMOS $/ 10 \mathrm{mg}$ HMDS
\end{tabular}

${ }^{a}$ Alongside $20 \mathrm{mg}$ EPMM and EtPDMS, each, and double the cuing duration.

present study. Labels were provided according to the amounts of both silylating agents, HMDS and TMOS, within the modified coatings as presented in Table 1. The synthesis schematics for these silylated superhydrophobic siloxane/PDMS hybrid nanocomposite coatings and their fabrication scheme for fabric surface modification are summarized in Fig. 1.

\subsection{Preparing fabric surfaces modified with silylated silica nanocomposite coatings}

Prior to fabric surface modifications, clean cotton fabrics $(5 \times 5$ $\mathrm{cm}$ ) were prewashed with ethanol, acetone and deionised water, separately, through ultrasonication (Branson M1800). This procedure was repeated three times in order to completely remove adhering surface contaminants and those embedded within the cotton fabrics. These fabrics were then dried at $30{ }^{\circ} \mathrm{C}$ for $15 \mathrm{~min}$ and then stored up for further use. They appeared clean with characteristic almost crispy feel when immediately removed from the vacuum oven, however, their physical forms were unaltered from this thermal treatment process. These superhydrophobic coatings were separately stirred with each fabric completely immersed within them at $30{ }^{\circ} \mathrm{C}$ for $3 \mathrm{~h}$ and ultrasonicated for $1 \mathrm{~h}$. This ended the surface modification process. As represented in Fig. 1, the coated fabrics were removed at a $200 \mathrm{~mm} \mathrm{~min}^{-1}$ withdrawal speed (KSV NIMA Dip Coater). This coating technique represents a low cost and reliable method for depositing wet coating films by simply immersing the solid fabric substrates within the coating suspension and withdrawing them at constant speed. This was closely followed by surface curing of coated fabrics using vacuum drying (Thermo Scientific Vac Oven $12.5 \mathrm{~L}$ ) at $40{ }^{\circ} \mathrm{C}$ for $1 \mathrm{~h}$ in order to remove unreacted and excess solvents on the fabric surfaces. They were also labelled accordingly and stored under a desiccator tor for further use.

\subsection{Characterization of silylated superhydrophobic coated fabric filters}

Field emission scanning electron microscopy (Hitachi SU6600 SEM) was utilized in analyzing the surface morphologies of both uncoated and all coated fabrics at appropriate acceleration voltage $(10-20 \mathrm{kV})$. Functional group chemistries of adhering

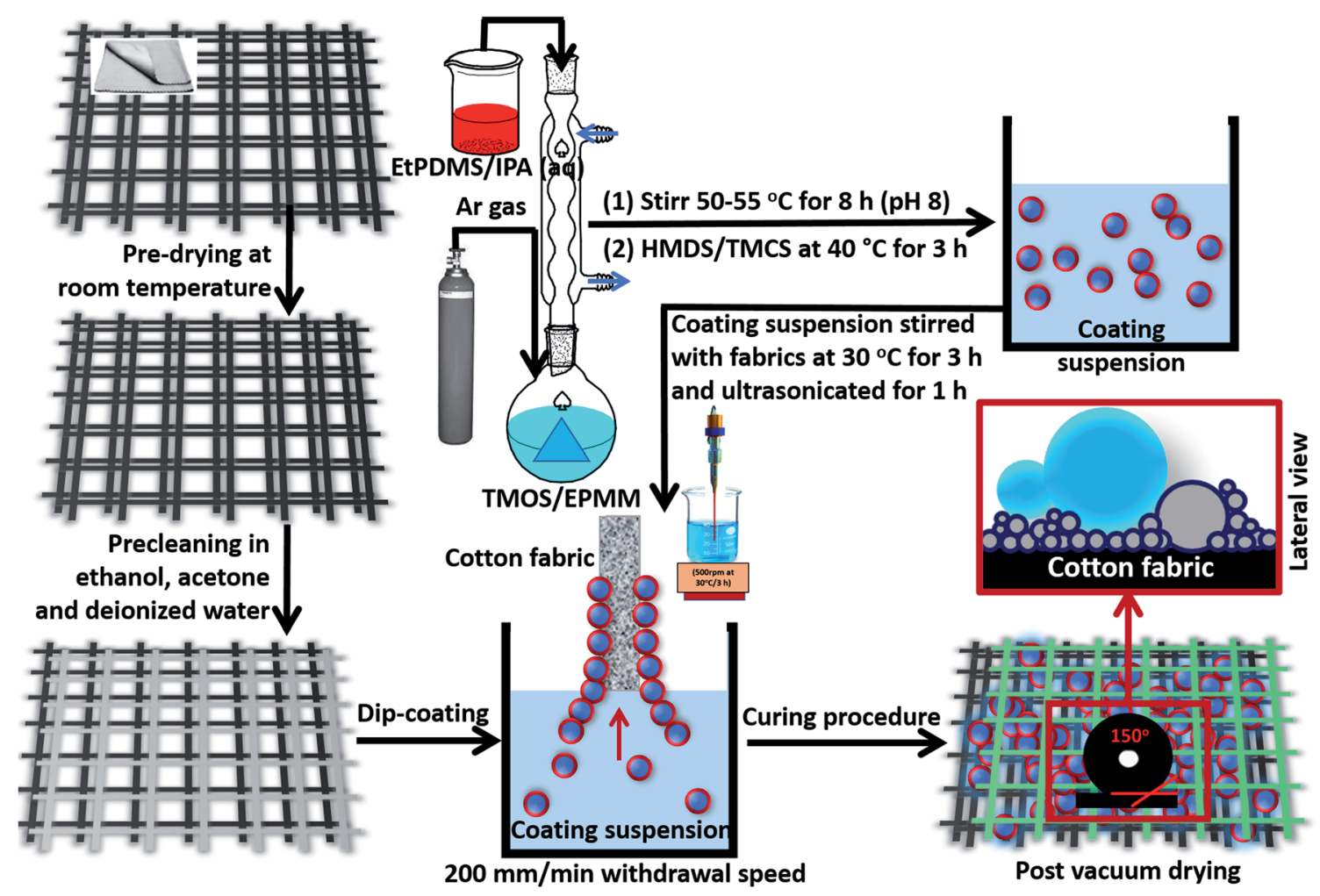

Fig. 1 An annotated schematic showing the preparation procedure for silylated superhydrophobic siloxane/PDMS hybrid nanocomposite coatings on cotton fabrics reported in this study. The process starts from the actual synthesis of silylated superhydrophobic coatings, precleaning procedure of all cotton fabric substrates and surface modification of precleaned cotton fabrics by dip-coating technique. 
coatings on the fabrics were also probed by means of Fourier Transform Infrared (FTIR) spectroscopy and X-ray photoelectron spectroscopy (XPS). These techniques also accounted for the degree of chemical conversions between reaction precursors and the resulting coating composite products for each synthesis step. FTIR spectroscopic analyses were conducted using a Renishaw Invia Reflex Raman microscope (with FTIR) equipped with an IlluminatIRII FTIR microscope accessory (Smith's Detection, Danbury, CT). Spectral plots were recorded at a $4 \mathrm{~cm}^{-1}$ spectral resolution between 400 and $4000 \mathrm{~cm}^{-1}$ wavelength range with a system that uses a $36 \times$ diamond ATR objective (10-50 $\mu \mathrm{m}$ aperture). The Invia Reflex used was a $514.5 \mathrm{~nm}$ laser excitation. X-ray Photoelectron Spectroscopy (XPS) analyses of both uncoated and coated fabrics were conducted using Kratos AXIS Supra XPS; all coated material surfaces were compatible with its ultra-high vacuum (UHV). The instrument is equipped with an $\mathrm{Al} \mathrm{K} \alpha$ monochromatic X-ray source. All tests were carried out at a $90 \mathrm{deg}$ on 120 micron spot size. X-ray scans for high resolution measurements proceeded using $0.05 \mathrm{eV}$ steps with pass energy, $15 \mathrm{keV}$ accelerating voltage and 15 $\mathrm{mA}$ emission current, respectively. The present study depended on evaluating inherent changes on the coated surfaces after various treatments. Here, the aqueous contact $\left(\theta_{\mathrm{w}}^{\circ}\right)$ and sliding hysteresis $\left(\theta_{\mathrm{H}}^{\circ}\right)$ angles of respective coated fabrics were measured using Data Physics contact-angle meter. This was measured after carefully placing approximately $5 \mu \mathrm{L}$ tiny deionized water drops on each fabric surface at ambient temperature. The presented results in this study are mean value of five measurements.

\subsection{Tests for chemical stability and self-cleaning abilities of superhydrophobic cotton fabric filters}

These silylated superhydrophobic coated cotton fabrics were completely exposed to $50 \mathrm{~mL} \mathrm{HCl}$ and $\mathrm{NaOH}$ (one molar each) for $24 \mathrm{~h}$ in order to test their chemical stabilities. After each test within respective solvents, each fabric was repeatedly rinsed with absolute ethanol, dried in vacuum for $1 \mathrm{~h}$ at $35^{\circ} \mathrm{C}$ before measuring its surface aqueous contact angles. Similar tests were also conducted after exposure to defined thermal conditions in vacuum between 40 and $70{ }^{\circ} \mathrm{C}$. The self-cleaning ability of the best performing superhydrophobic coated fabric was also examined after placing finely ground powders of colored chalks. This coated fabric was placed on glass slide with equal tilt angle in Petri dishes alongside an uncoated fabric. Water droplets were then carefully introduced from a Diamond ${ }^{\mathrm{TM}} \mathrm{Jr}$ Mini Pipettor (Globe Scientific) and allowed to roll through these fabric surfaces while monitoring the extent of wetness and powder/dirt accompanied roll-off.

\subsection{Oil-water separation with superhydrophobic cotton fabric filters}

The percentage separation efficiencies of these silylated superhydrophobic coated fabrics were also determined for oil-water mixtures. Here, the coated fabrics were utilized as separation membranes in a filtration test involving repeated cycles after individually fitted appropriately to a laboratory-fabricated filtration set-up. The fabrics were firmly secured between the volumetric glass and the round bottom flask in order to allow the filtrate pass through to the flask without spillage while also retaining the reduce. To simulate a reliable oil-water mixture, chloroform, toluene and water samples were utilized in this test. Chloroform and toluene were separated individually from $50 \mathrm{~mL}$ of each oil-water mixture per cycle. Firstly, chloroform acted as the heavy oil phase mixed with an aqueous (i.e., water) phase. This chloroform-water mixture was allowed to pass through the setup and separated accordingly after pouring it through a funnel. Since both liquids are colorless and almost very difficult to observe the separation of both phases within the setup, it was pertinent stain one of them with a dye. By staining water with $5 \mathrm{wt} \%$ methyl orange, the aqueous phase possessed a near orange coloration, allowing for a distinct chloroform-
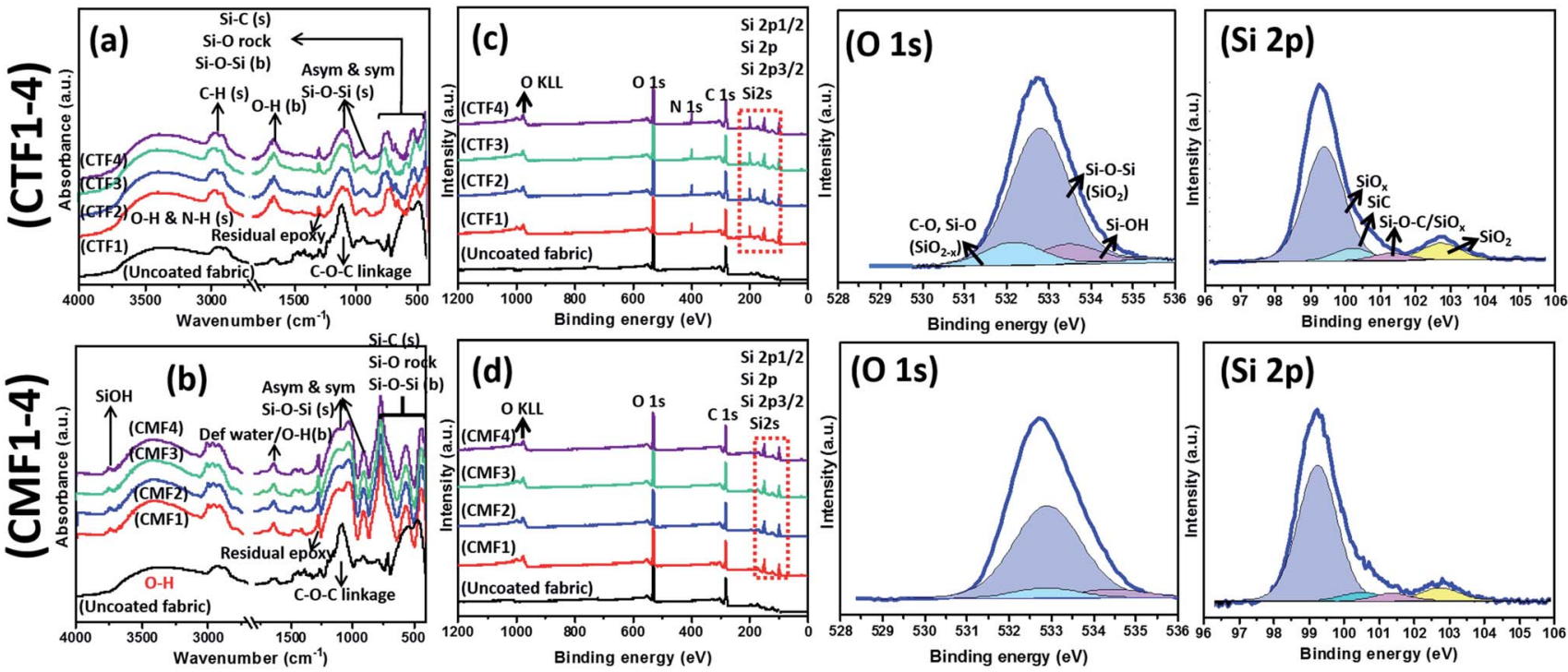

Fig. 2 FTIR $(a, b)$ and XPS wide scan (c, d) and deconvoluted high-resolution $O$ 1s and Si $2 p$ curves of coated (CTF1 and CMF1-4) superhydrophobic fabrics. 
water separation toward fostering a good separation efficiency. Methyl orange was soluble only in water and not chloroform, hence, creating a distinct separation boundary between the heavy organic (chloroform) and light aqueous phases. The dyed water remained above membrane due to the superhydrophobicity of the coated fabric filter. However, chloroform filtered through and settled at the bottom of flask by means of gravitational force since it is also heavier than water. This filtration test was conducted for five cycles after rigorous shaking of the chloroform-water mixtures before filtration. This test was also conducted for a dye-stained lighter organic phase (toluene) in combination with water for the same number of cycles and mixture volume (50 mL each cycle). For both toluene/ water and chloroform/water mixtures, the separation efficiencies (\%) of the superhydrophobic cotton fabrics were computed using eqn (1). ${ }^{4}$

Percentage separation efficiency $(\%)=$ $\frac{\text { mass of collected oil after filtration }}{\text { initial mass of added oil before filtration }} \times 100$

\section{Results and discussion}

\subsection{Compositional analyses of superhydrophobic coated} fabrics

Compositional analyses of bare and respective coated superhydrophobic fabrics were conducted using FTIR and XPS techniques. The FTIR spectra of CTF1-4 and CMF1-4 coated fabrics are depicted in Fig. 2(a and b). There is a strong adsorption band at $1030 \mathrm{~cm}^{-1}$ consistent with symmetric and asymmetric glycosidic linkage stretching COC vibrations and few other cellulose functional groups overlap (e.g., C-C and C-O) (see the FTIR spectrum of the uncoated fabric). There are also broad stretching bands ( $\mathrm{H}$ connected) from an $\mathrm{O}-\mathrm{H}$ vicinal between $3000-3600 \mathrm{~cm}^{-1}$ corresponding to silanol $n(\mathrm{Si}-\mathrm{OH})$ groups. ${ }^{18}$ Both peaks are also present on the spectra of coated fabrics with terminal silanols and $\mathrm{C}-\mathrm{H}$ stretching (from $\mathrm{Si}-\mathrm{CH}_{x}$ alkyl groups) vibrations observed at 3750 and $2976 \mathrm{~cm}^{-1}$ shoulders for CMF1-4 compared to CTF1-4. More conspicuous on the spectra of coated fabrics are symmetric and asymmetric $\mathrm{Si}-\mathrm{O}-\mathrm{Si}$ stretching vibrations at 877 and 1044/1086 $\mathrm{cm}^{-1}$, respectively. These peaks could be attributed to the formation of polysiloxane network after sol-gel induced condensation reactions from siloxane precursors. ${ }^{\mathbf{1 9} 20}$ There are $\mathrm{Si}-\mathrm{O}-\mathrm{Si}$ bending stretching and $\mathrm{Si}-\mathrm{O}$ rocking vibrations between 400 and $430 \mathrm{~cm}^{-1}$ for both sets of coated fabrics. The presence of $\mathrm{Si}-\mathrm{C}$ stretching peaks at $1400 \mathrm{~cm}^{-1}$ could be attributed to long octadecyl and methyl moieties on coating matrices for CMF1-4 and CTF1-4, respectively. ${ }^{19,21}$ It is the presence of these unreactive organic alkyl groups that contributes to the superhydrophobicity of these siloxane/PDMS silicon hybrid elastomer coatings on the fabrics. Adhering $-\mathrm{CH}$ groups on the coated fabrics significantly contribute to their low wetting capacity due to the lowered surface-energy PDMS additive after coating modification. ${ }^{4}$ There are also residual ring-breathing vibration peaks of epoxy at $1255-1260 \mathrm{~cm}^{-1}$.

XPS wide scan spectra of respective superhydrophobic coated fabrics are also depicted in Fig. 2(c and d) relative to the

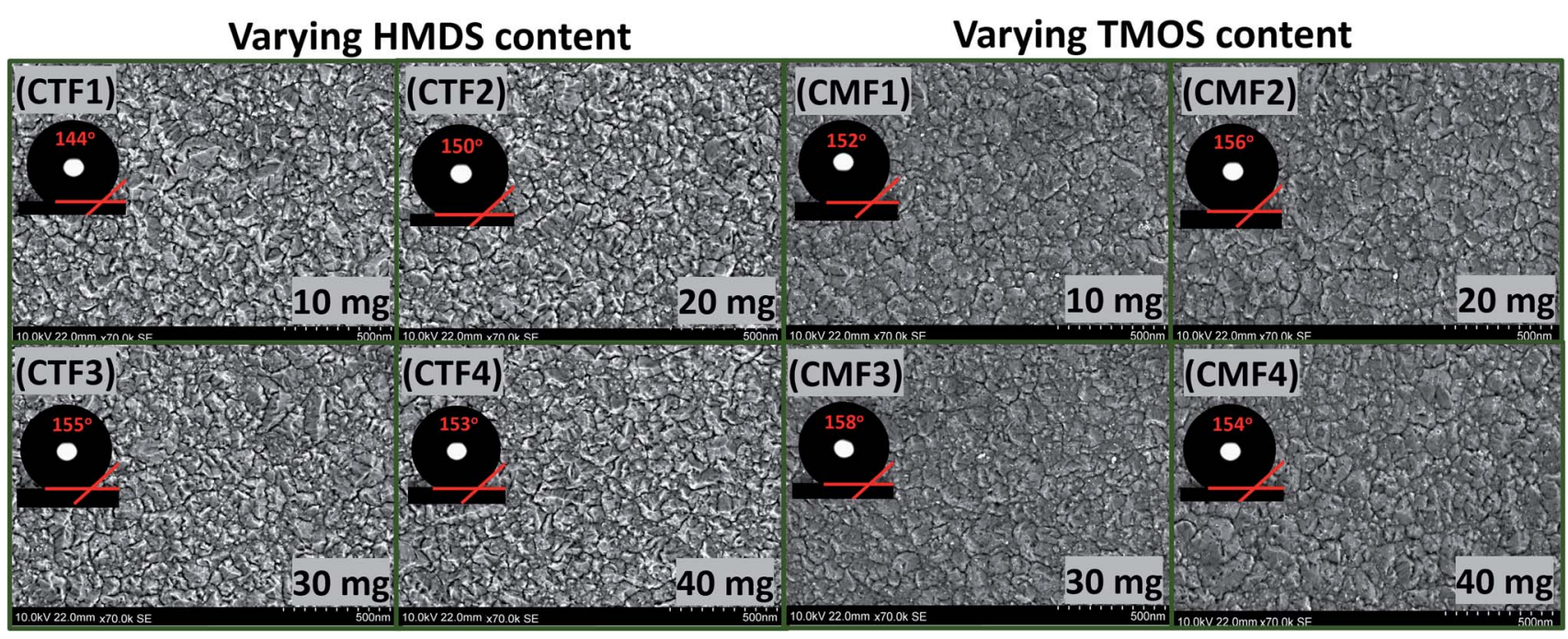

Fig. 3 High-magnification FESEM micrographs of superhydrophobic coated cotton fabrics with varying HMDS and TMOS contents.

Table 2 Individual weights of cotton fabrics substrates $(5 \times 5 \mathrm{~cm})$ before any treatment

\begin{tabular}{llllllllll}
\hline Cotton fabric & Bare fabric & CTF1 & CTF2 & CTF3 & CTF4 & CMF1 & CMF2 & CMF3 & CMF4 \\
\hline Weight $(\mathrm{g})$ & 10.5 & 14.1 & 19.8 & 21.2 & 22.5 & 14.2 & 20.8 & 22.8 & 25.7
\end{tabular}


uncoated substrate. While there are few peaks consistent with $\mathrm{C}$ and $\mathrm{O}$ atoms at binding energies of 286.5, 534.4 and $984.6 \mathrm{eV}$, respectively, there are also core level peaks consistent with the major Si coating components at 97.8 and $150.0 \mathrm{eV} .^{22}$ There are no Si XPS peaks on the bare fabric while the only distinct difference between CMF1-4 and CTF1-4 is in the N peak at $400 \mathrm{eV}$. Deconvoluted high-resolution spectra for O 1s and Si 2p from coated (CTF1 and CMF1-4) superhydrophobic fabrics are also presented on Fig. 2. The following bonds are identified on the deconvoluted $\mathrm{O}$ spectra as $\mathrm{O}$ 1s peaks: $\mathrm{Si}-\mathrm{O}-\mathrm{Si}$ from $\mathrm{SiO}_{2}$ (533.1 eV), Si-O (531.9 eV) and $\mathrm{Si}-\mathrm{OH}(534 \mathrm{eV}) .{ }^{23}$ The observed peaks near $523 \mathrm{eV}$ are related to $\mathrm{O}$ atom vacancies at the $\mathrm{Si}$ surface ${ }^{\mathbf{2 0 , 2 1}}$ due to changes in mobility of the $\mathrm{SiO}_{2-x}$. Meškinis et $a .^{23}$ also pointed out that these peaks may also be linked with $\mathrm{SiOC} / \mathrm{SiO}_{2} / \mathrm{SiO}_{x}$ and silanol $(\mathrm{Si}-\mathrm{OH})$ at approximately, 95\% and $5 \%$, respectively. The wide spectra show lower pass energy consistent with fused Si 2 p peaks $\left(2 \mathrm{p}_{1 / 2}\right.$ and $\left.2 \mathrm{p}_{3 / 2}\right)$ at $100.8 \mathrm{eV}$ (and $\mathrm{Si} 2 \mathrm{~s}$ at $152.86 \mathrm{eV}$ on the wide scan spectra) due to the amorphous siloxane/PDMS phase on both coatings. ${ }^{24}$ There are four designated peaks corresponding to $\mathrm{SiO}_{x}, \mathrm{SiC}, \mathrm{Si}-\mathrm{O}-\mathrm{C}$, and $\mathrm{SiO}_{2}$ bonds on the $\mathrm{Si}$ 2p spectra on both coatings between 99.6 and $103.5 \mathrm{eV} .^{23,25}$ The SiC peak is an overlap between $\mathrm{Si}-\mathrm{C}$ and $\mathrm{Si}-\mathrm{O}_{x}$ bonds. Both XPS Si 2p and $\mathrm{O}$ 1s spectra reveal the presence of $\mathrm{SiO}_{2-x}$ and $\mathrm{Si}-\mathrm{OH}$ states on both coatings, which is also confirmed by FTIR spectra. These XPS results confirms the presence of polysiloxane chemical groups within the surface nanostructures of the fabrics.

\subsection{Surface morphology of silylated superhydrophobic coated fabrics}

The low- and high-magnification SEM images (from left to right) showing the surface morphologies of bare and coated superhydrophobic fabrics are depicted within the ESI (Fig. S1). $\dagger$ The uncoated fabric displayed a flat morphology with no distinct microfeature. However, there were significant changes in this rather smooth fabric surface after modification with superhydrophobic coatings as displayed on high magnification. These nanocomposites appear to be evenly distributed on individual fabric fibers. SEM image of the coated fabric also reveals a unique morphology with evenly sparse but with rather rough nanoporous structures. It is also worthy of note to mention that variants of this superhydrophobic coating were further prepared according to varying concentrations of the PDMS (10-40 mg). In this study, all coated cotton fabrics were labelled CTF1-4 (10-40 mg HMDS/10 mg TMOS) and CMF1-4 (10-40 $\mathrm{mg}$ TMOS/10 $\mathrm{mg}$ HMDS) according to the HMDS and TMOS contents. Fig. 3 shows the SEM micrographs of coated superhydrophobic fabric at higher magnifications. The surfaces of these coated fabrics possess numerous porous nanostructures with protrusive surface architectures responsible for trapping air within them, thereby creating a hydrophobic resistance against water penetration. These nanopatterned surface grooves further reduced gross surface energy as the coating morphology contributes to the formation of more air nanobubbles traps within the coatings. ${ }^{4}$ Compared to the bare fabric, the coated fibers showed significant porous nanostructures while their morphologies show distinct nanohierarchical roughness that also contributes to superior static contact angles. This present study reveals a one-pot approach technique for fabricating a nonfluorinated hybrid organicinorganic silica nanocomposite coated morphology synthesized after trimethylsilyl modification with hexamethyldisilazane (HMDS) and trimethoxy(octadecyl)silane (TMOS) silylating agents. The presence of these silylating agents promoted enhanced superhydrophobicity when deposited on cotton fabric. It is also worthy of note to mention that coated fabrics were heavier compared to the bare fabric substrate. The differences between CTF1-4 and CMF1-4 could be due to their

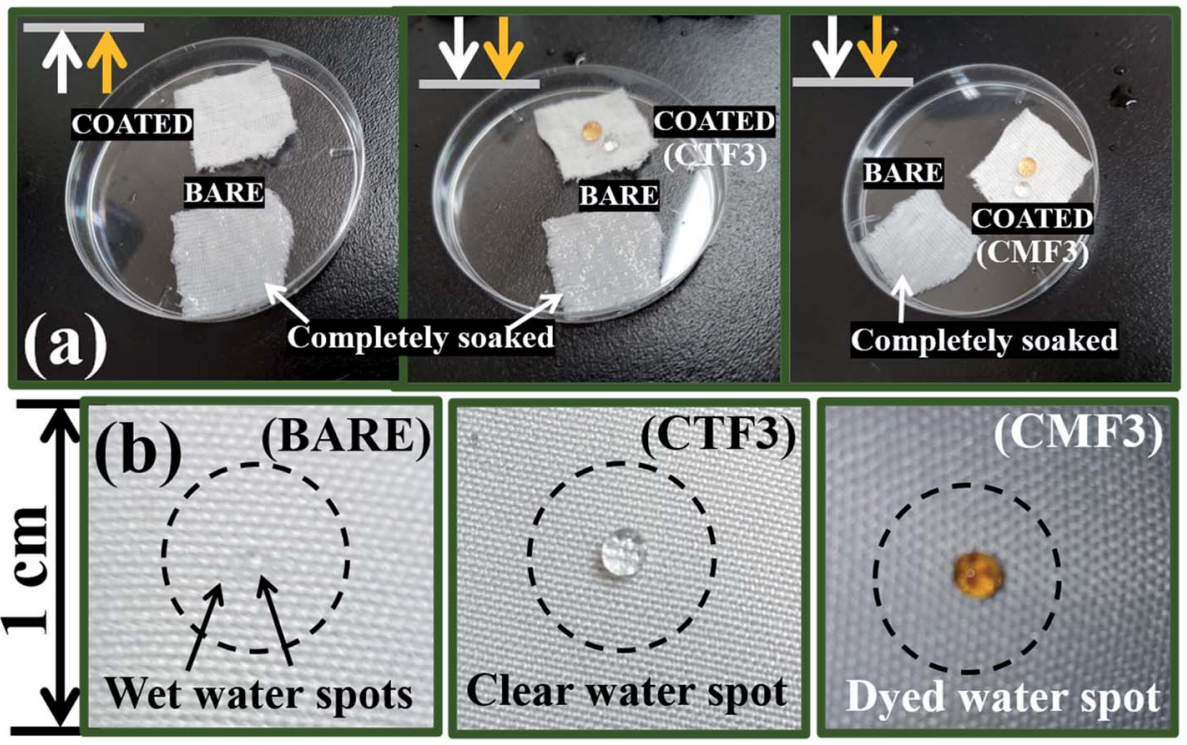

Fig. 4 Normal (a) and magnified (b) photographic images showing the differences in surface wettability between bare and superhydrophobic coated cotton fabrics when placed on pure and dyed water droplets (arrows up) and vice versa (arrows down). The colour of cotton fabrics in this study is off-white; the observed appearances could be due to varying light shades in the laboratory. 
respective silica contents. The measured weights of all cotton fabrics before any treatment are presented in Table 2 . In both coating systems, higher fabric weights were observed with increasing silylating agents (10-40 mg HMDS and TMOS). Preliminary study revealed unique superhydrophobic properties for both sets of coatings. Fig. 4 shows the differences in surface wettability between bare and superhydrophobic coated cotton fabrics when they were placed on pure and dyed water droplets and vice versa.

\subsection{Examining the effects of silylating coating contents on the wetting behaviors of coated fabric filters}

The ability of these coated surfaces to self-clean depends on their hydrophobicity. Specifically, the contact angle of water on surfaces of this kind represents an important characteristic that also determines the capacity of a coated surface to self-clean. Contact angle is influenced by the microstructural surface roughness, and the Young, Wenzel and Cassie-Baxter's models (eqn (2)) have been developed to describe inherent wettability of self-cleaning surfaces. While the Young's model describes the droplet-surface relations on perfect surfaces, the other two models account for interface effects from rough surfaces. Specifically, the Cassie-Baxter's model of wetting recounts for changes at the interface when adhering water droplets creates air pockets at these surfaces.

$$
\begin{aligned}
\cos \left(\theta_{\mathrm{o}}\right) & =\left(\gamma_{\mathrm{SA}}-\gamma_{\mathrm{SL}} / \gamma_{\mathrm{LA}}\right) ; \cos (\theta)=R_{\mathrm{f}} \cos \left(\theta_{\mathrm{o}}\right) ; \cos \left(\theta_{\mathrm{CB}}\right) \\
& =R_{\mathrm{f}} \cos \left(\theta_{\mathrm{o}}\right)-f_{\mathrm{LA}}\left(R_{\mathrm{f}} \cos \left(\theta_{\mathrm{o}}\right)+1\right)
\end{aligned}
$$

Before examining the effects of silylating coating contents on the collective wetting behaviours of these coated fabric systems, a preliminary water-droplet test was conducted by placing (i) multiple drops of water on exposed coated fabric surfaces and (ii) also by placing these coated surfaces on water spills. The extent of water adsorption is indicative to the hydrophobicity of these fabrics (see Fig. 4(a)). Corresponding photographic images showing the differences in surface wettability between bare and superhydrophobic coated cotton fabrics are also depicted in Fig. 4(b), for both clear and dyed water droplets. These water droplets sat on the coated fabric surfaces without wetting them. However, the bare fabrics were soaked up after the droplets spread on the surface. The initial contact angle measurements both sets of coated fabrics revealed values up to $150^{\circ}$, denoting superhydrophobicity.

Fig. 5 depicts the variation of aqueous contact $\left(\theta_{\mathrm{w}}^{\circ}\right)$ and sliding hysteresis $\left(\theta_{\mathrm{H}}^{\circ}\right)$ angles of coated fabrics. The trend of results reveals consistent influence of silylated coating contents on surface wettability. Surface hydrophobicity increased with HMDS content (10-40 mg HMDS) while TMOS concentration kept unchanged $(10 \mathrm{mg}$ ) within the coating. The coated fabrics in this study demonstrated significant superhydrophobicity at values of static $\theta_{\mathrm{w}}^{\circ}$ more than $150^{\circ}$, except for CTF1 with $10 \mathrm{mg}$ HMDS which also recorded the highest contact angle hysteresis $\left(20^{\circ} \pm 0.5^{\circ}\right)$. Fig. 5(a) reveals increase $\theta_{\mathrm{w}}^{\circ}$ values in the order: CTF3 $\left(155.9^{\circ} \pm 0.6^{\circ}\right)>\mathrm{CTF} 2\left(150.4^{\circ} \pm 0.5^{\circ}\right)>\mathrm{CTF} 1\left(144.5^{\circ} \pm\right.$ $0.7^{\circ}$ ) with 30,20 and $10 \mathrm{mg}$ HMDS, respectively. Other than CTF4 $\left(153.5^{\circ} \pm 0.7^{\circ}\right)$, increment in HMDS content within the coating significantly improved the superhydrophobicity of the fabric surface. CTF3 demonstrated superior protection with recorded magnitudes of values of $\theta_{\mathrm{w}}^{\circ}$ and $\theta_{\mathrm{H}}^{\circ}$ up to $155.9^{\circ} \pm 0.6^{\circ}$ and $13.8^{\circ} \pm 0.3^{\circ}$. With HMDS, the rough and porous nanostructures of the coated surface was enriched with stable substituted silyl $\left(\mathrm{R}_{3} \mathrm{Si}\right)$ groups. These features contributed to improved hydrophobicity, beyond which the surface fabric wettability increased, and this could have been due to saturation of the nanocomposite coating pores with HMDS. At this stage, these surface change had become unfavorable toward the formation trapped air within nanostructure of the coating, hence, disrupting Cassie-Baxter surface tension between the sitting liquid drop and the surrounding air., ${ }^{2,4}$

To investigate the effect of TMOS content on surface wettability, bare fabrics were modified with coatings containing 10-
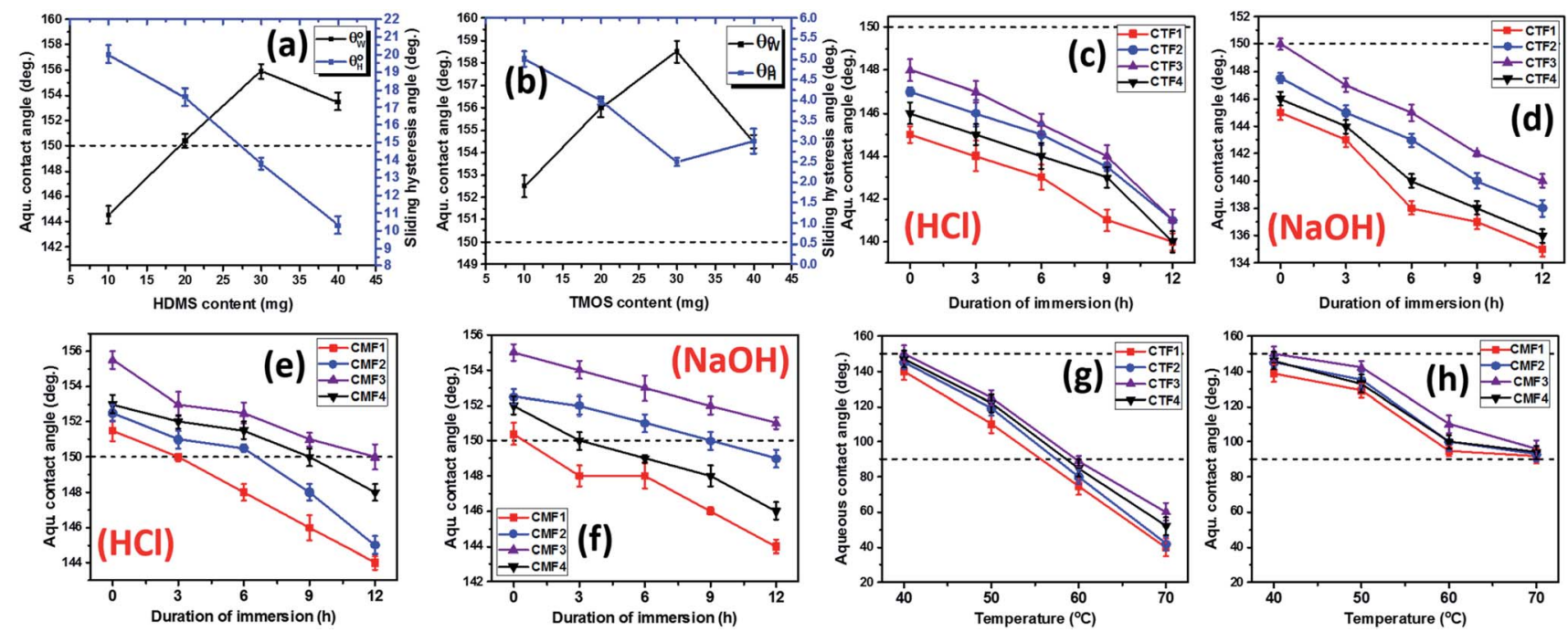

Fig. 5 The wetting behaviors of cotton fabrics with varying HMDS (a) and TMOS (b) silylating coating contents without treatments. The effects of corrosive $\mathrm{HCl}(\mathrm{c}, \mathrm{e})$ and $\mathrm{NaOH}(\mathrm{d}, \mathrm{f})$ solvents and varying thermal conditions ( $\mathrm{g}, \mathrm{h}$ ) on the surface wettability of coated superhydrophobic cotton fabrics. 


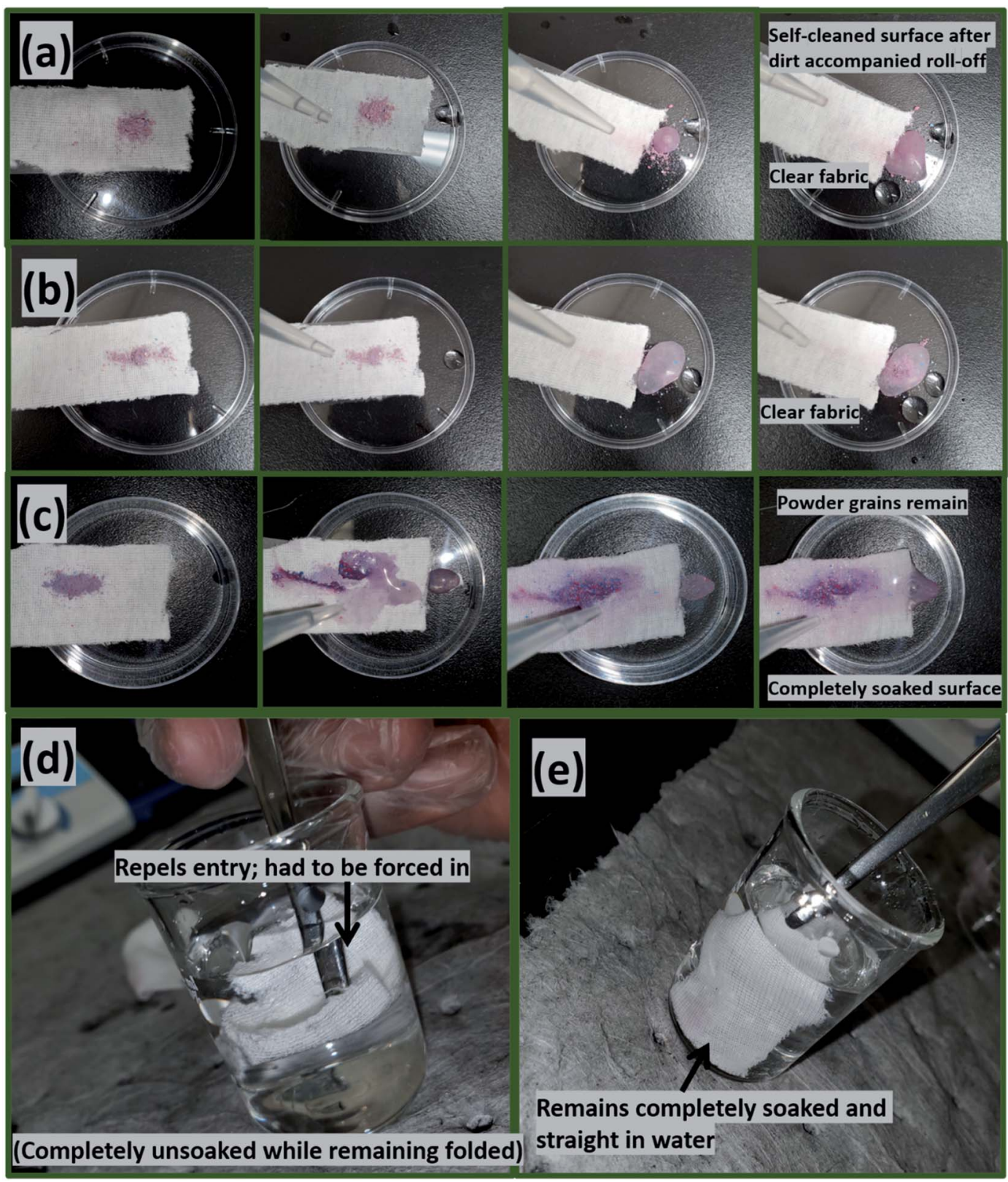

Fig. 6 Optical images showing varying wetting behaviours of self-cleaning coated superhydrophobic fabrics (a, CTF3 and b, CMF3) compared to bare fabric (c). The wetting behaviours of coated superhydrophobic (d) and bare (e) fabrics during complete submersion in water.

$40 \mathrm{mg}$ TMOS mixed with a fixed HMDS concentration $(10 \mathrm{mg})$; labelled CMF1-4. The magnitudes of surface contact angle $\left(\theta_{\mathrm{w}}^{\circ}\right)$ were also measured. The trend of this parameter noticeably increased with TMOS content within the coated fabric up to $30 \mathrm{mg}$ (being the optimum concentration of silylating agent offering the most superhydrophobicity). Compared to CMF3, the value of $\theta_{\mathrm{w}}^{\circ}$ for CMF4 was less due to overpacked coating network with silica/PDMS nanocomposite with stable nonhydrolysable octadecyl moieties. From Fig. 5(b), higher $\theta_{\mathrm{w}}^{\circ}$ values $\left(158.5^{\circ} \pm 0.5^{\circ}\right)$ were recorded for CMF3 with $30 \mathrm{mg}$ TMOS while the least was recorded for $10 \mathrm{mg}$ TMOS $\left(152.5^{\circ} \pm 0.5^{\circ}\right)$. The aqueous rolling angle was also observed to reduce with TMOS content; the least and most recorded magnitudes of $\theta_{\mathrm{H}}^{\circ}$ were $2.5^{\circ} \pm 0.1^{\circ}$ for CMF3 and $5.0^{\circ} \pm 0.2^{\circ}$ for CMF1. CMF1-4 coated fabric exhibited superior superhydrophobicity compared to CTF1-4 since their surfaces still maintained inherent superhydrophobicity with values of $\theta_{\mathrm{w}}^{\circ}$ greater than $150^{\circ}$ as well as values of $\theta_{\mathrm{H}}^{\circ} \leq 5^{\circ}$. The contribution of silylation to surface wetting mechanism for CMF1-4 and CTF1-4 coated fabrics is presented within the ESI (Fig. S2) $\cdot \dagger$

\subsection{Examining the chemical and thermal stabilities of} silylated superhydrophobic coated fabric filters

Since the coated fabrics would be utilized is various service environments with several inherent factors capable of affecting their protective performances, ${ }^{26}$ stability tests were conducted in $1 \mathrm{M} \mathrm{HCl}$ and $\mathrm{NaOH}$. These tests involved the complete immersion of the coated fabrics in $50 \mathrm{~mL}$ test solutions for $24 \mathrm{~h}$. 
After each test within respective solvent, each fabric was repeatedly rinsed with absolute ethanol, dried in vacuum for $1 \mathrm{~h}$ at $35{ }^{\circ} \mathrm{C}$ before measuring its surface aqueous contact angles. Fig. 5(c-f) depicts the trend of $\theta_{\mathrm{w}}^{\circ}$ values after exposure to $\mathrm{HCl}$ and $\mathrm{NaOH}$ solvents. In both corrosive media, CTF1-4 coated fabrics performed less than CMF1-4 as observed in the decreased magnitudes of $\theta_{\mathrm{w}}^{\circ}$. Unlike CTF1-4, CMF1-4 coated fabrics still retained their superhydrophobicity to some extent,
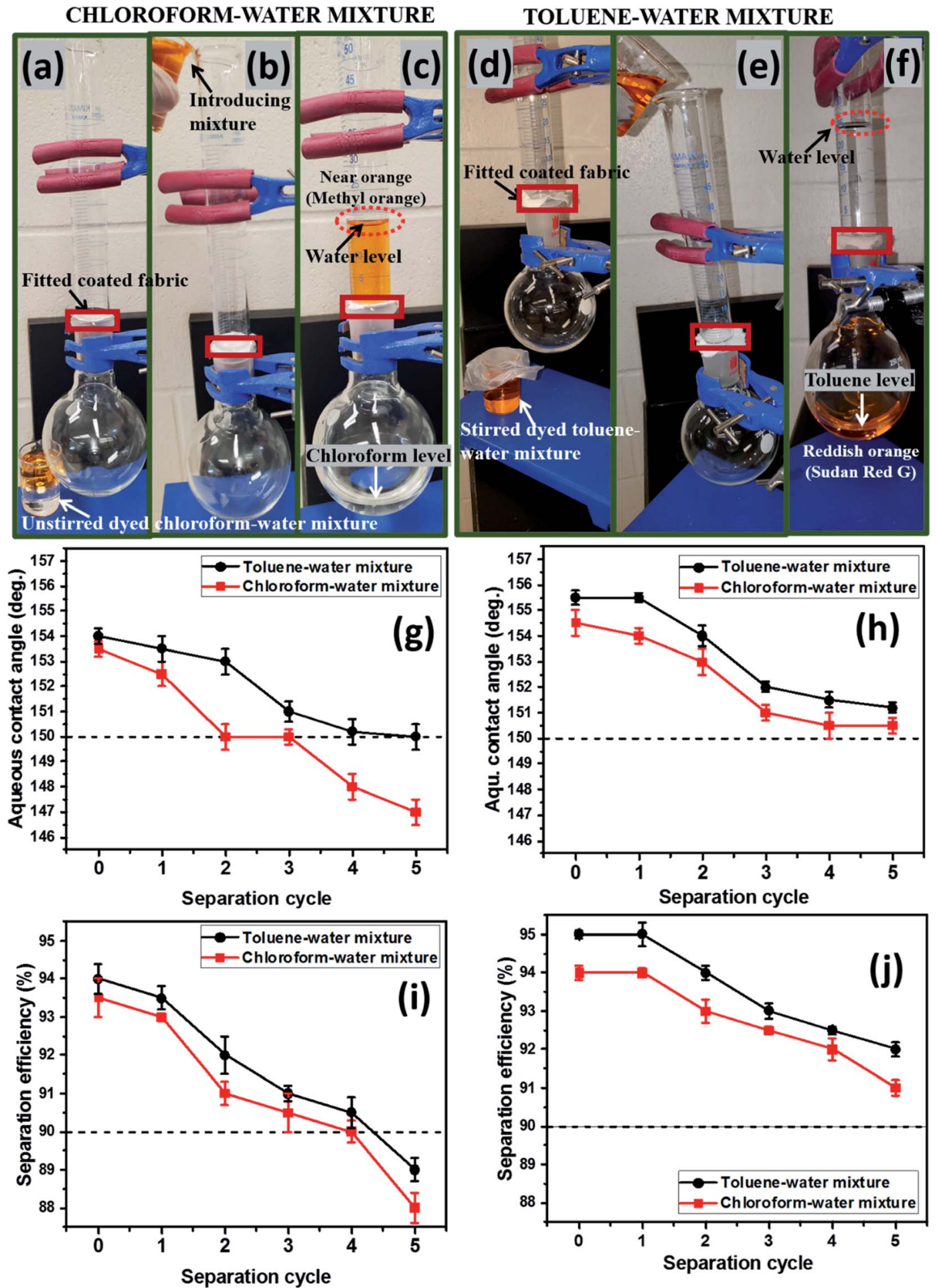

Fig. 7 Laboratory fabricated oil-water filtration setups showing ( $a$, d) fitted silylated superhydrophobic CMF3 coated fabric alone; (b, e) introduction of the heavy oil-water and light oil-water mixtures prior to the filtration process; (c, f) completely separated oil and water after several filtration cycles. ( $g, h)$ Aqueous contact angle fluctuations and (i, j) separation efficiency for the best coated superhydrophobic fabrics (left: CTF3 with $30 \mathrm{mg} \mathrm{HMDS} / 10 \mathrm{mg}$ TMOS and right: CMF3 with $30 \mathrm{mg}$ TMOS/10 mg HMDS) between 0 to 5 separation cycles. 
hence their superior protective properties, since their $\theta_{\mathrm{w}}^{\circ}$ values stood above $150^{\circ}$. The reason for this could be linked with influences of the long octadecyl C18 moiety from the trimethoxy(octadecyl)silane (TMOS) precursor that contributed to the stable superhydrophobic character of these coated fabric relative to HMDS modification. ${ }^{27}$ However, values of $\theta_{\mathrm{w}}^{\circ}$ significantly reduced less than $150^{\circ}$ for coated fabrics with lesser TMOS content (CMF1-2 with 10 and $20 \mathrm{mg}$ TMOS and $10 \mathrm{mg}$ HMDS) between 0 and $12 \mathrm{~h}$ due to the corrosive impact of both solvents $(\mathrm{HCl}$ and $\mathrm{NaOH})$. Though both solvents impacted on these coated fabrics, $\mathrm{NaOH}$ had the most effect relative to $\mathrm{HCl}$. In both solvents, higher performances were recorded for coated fabrics with $30 \mathrm{mg}$ TMOS/10 $\mathrm{mg}$ HMDS (CMF1) as well as $30 \mathrm{mg}$ HMDS/10 mg TMOS (CTF3) due to their unique surface chemistry and contribution of their nanostructures. Normally, the polysiloxane networks within most coatings of this nature show significant chemical resistance.

In the test for thermal stability, all coated fabrics were exposed to defined thermal conditions in vacuum between 40 and $70{ }^{\circ} \mathrm{C}$ for an hour before measuring their magnitudes of $\theta_{\mathrm{w}}^{\circ}$. The results of thermal stabilities of these silylating superhydrophobic coated fabrics are presented in Fig. 5( $\mathrm{g}$ and $\mathrm{h}$ ). Between both coating systems, there was significant reduction in superhydrophobicity due to the impact heat. This must have led to loss of protective performance due to breakdown of polymer chains and loss of coating-fabric adhesion. ${ }^{28,29}$ However, CMF1-4 coated fabrics still exhibited hydrophobicity $\left(\theta_{\mathrm{w}}^{\circ}>90^{\circ}\right)$ compared to CTF1-4 due to increased silica content. The observed decrease in $\theta_{\mathrm{w}}^{\circ}$ could be linked with pyrolysis of their organic PDMS phase and it was more for CTF1-4 than CMF1-4. At $70{ }^{\circ} \mathrm{C}$, there was complete degradation of these surface CTF1-4 coating, however, CTF4 still showed higher $\theta_{\mathrm{w}}^{\circ}$ magnitudes. Intense heat could have also contributed to loss of fabric bound hydroxyl chemical groups beneath these coated. ${ }^{4}$ Coatings with higher TMOS contents showed more surface stability due to the low thermal expansion and high thermal stability of the sol-gel derived polysiloxane coating network ( $n \mathrm{Si}-\mathrm{O}-\mathrm{Si}$ ). The incorporation of this secondary phase contributed to stable thermal shock resistant and conductivity unlike the organic HMDS presence that only increased superhydrophobicity.

\subsection{Self-cleaning capacities of silylated superhydrophobic coated fabric filters}

In this section, the variants of coated fabrics with the best superhydrophobic performance were chosen; CTF3 (with $30 \mathrm{mg}$ HMDS/10 mg TMOS) and CMF3 (with $30 \mathrm{mg}$ TMOS/10 mg HMDS). To investigate the self-cleaning capacities of these coated fabrics, finely divided powdery grains of colored industrial chalks were placed on these coated fabrics. Fig. 6 depicts the optical images showing varying wetting behaviours of selfcleaning silylated superhydrophobic coated fabrics (a, CTF3 and b, CMF3) compared to bare fabric (c). These setups were then tilted at defined gradients on glass slides inserted in preclean Petri dishes before carefully introducing water droplets. Here, these droplets were allowed to slide through these powdery dust-leavened coated surfaces (a, b), in turn leaving clear paths of free of adhering dirt particles. The superhydrophobicity of these self-cleaned surfaces allowed for inherent removal of powdery debris. Conversely, Fig. 6(c) depicts significant surface wetness on the bare fabric after water adsorption. This hydrophilic character was due to the presence of cellulosic chemical groups on the fabric that also allowed for the adhesion of powdery dirt on the surfaces of this fabric without accompanied roll-off compared to the coated superhydrophobic fabrics. The reduced surface energies and higher hydrophobicity of coated surfaces contributed to the observed self-cleaning characters. This trend of results are direct contributions of incorporated silylated PDMS groups within these coated fabric surfaces. The whole fabric areas of both bare and coated fabrics showing their comparative wetting behaviours are depicted in Fig. 6(d). With the coatings, these non-wetted superhydrophobic fabrics quickly folded when forced into water and remained unsoaked due to creation of surface tension. This bright superhydrophobic coated surface remained unwetted in water as air is trapped within the roughened nanoand microporous fabric surfaces in water, in turn, leading to the reduced surface wetting. ${ }^{4}$ However, the bare fabric (Fig. 6(e)) rapidly adsorbed water, swelled and soaked up completely while it also remained straighten upon sinking unassisted into the bottom of the beaker. The observed surface property is consistent with superhydrophilicity when exposed to compared to coated fabrics under similar conditions.

\subsection{Using silylated superhydrophobic coated fabric filters as oil-water separation filters}

As presented in Fig. 7(a and d), the filtration setup fitted silylated superhydrophobic coated fabrics was utilized in oil-water separation. As modeled oil samples, chloroform (heavy oil) and toluene (light oil) were chosen due to their difference in densities when mixed with water. After introduction of both heavy oil-water and light oil-water mixtures prior to the filtration process $(b, e)$, separation of phases occurred according to the filtration cycle. In the case of chloroform, it separated and sank readily to the round-bottom flask as a colorless liquid, leaving the dyed aqueous phase on the upper glass over the coated silylated superhydrophobic fabric after several repeated filtration cycles (c, f). A similar test was also conducted for dyed toluene and this latter organic solvent also remained immiscible in water but less dense, so it floated above the aqueous layer. Summaries of the aqueous contact angle fluctuations $(\mathrm{g}$, h) of the best performed coated superhydrophobic fabrics (CTF3 with $30 \mathrm{mg}$ HMDS/10 mg TMOS and CMF3 with $30 \mathrm{mg}$ TMOS/10 mg HMDS) are depicted in Fig. 7. These results were collected after exposure to chloroform and toluene at defined separation cycles. In this study, chloroform was observed to be heavier than toluene while the water is denser than toluene. For both organic solvents, the coated cotton fabrics for this test retained their superhydrophobicity $\left(\theta_{\mathrm{w}}^{\circ} \geq 150^{\circ}\right)$ even after separation cycles under study, except for CTF3 (with $30 \mathrm{mg}$ HMDS/10 mg TMOS) after the fourth and fifth consecutive separation cycles in chloroform-water mixture. The least 


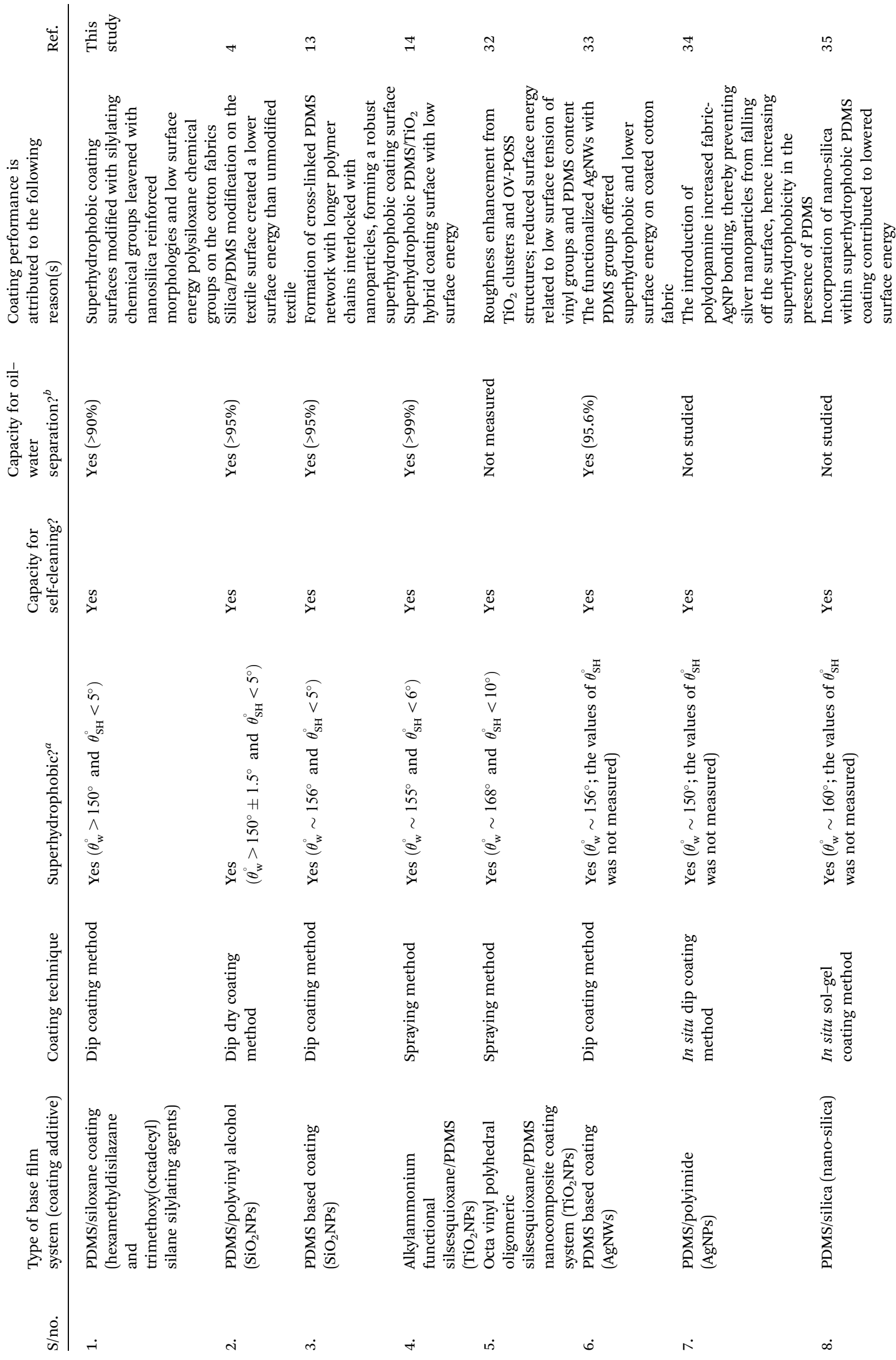


recorded values of $\theta_{\mathrm{w}}^{\circ}$ were $150.0^{\circ} \pm 1.5^{\circ}$ and $147.1^{\circ} \pm 0.5^{\circ}$ after the fifth filtration cycles in toluene and chloroform, respectively, for CTF3 while $151.1^{\circ} \pm 1.5^{\circ}$ and $150.5^{\circ} \pm 1^{\circ}$ were recorded for CMF3 at the same cycle. For maintaining contact angles up to $150^{\circ}$, these superhydrophobic coated fabrics demonstrated significant qualities for reusability and recyclability during filtration. Between both organic solvents, the surfaces of fabrics treated with chloroform were most impacted by the solvent treatments compared to toluene within the separation cycles under study. CMF3 remained the coated fabric with superior performance compared to CTF3 due to the formation of stable non-hydrolysable octadecyl moiety on nanosilica phases within the PDMS matrix at optimum trimethoxy(octadecyl)silane content. This alkyl moiety from the TMOS also provides the required low surface energy to the coating matrix needed to retain its superhydrophobicity. The computed values of chloroform-water and toluene-water separation efficiencies are presented in Fig. 7(i and j) between 0 and 5 cycles. In all, both silylated superhydrophobic coated fabrics retained separation efficiencies over 90\%. CMF3 recorded separation efficiencies of 94 and $95 \%$ for chloroform and toluene, respectively, while those of CTF3 stood at 88 and $89 \%$, in that order. The observed reduction in separation efficiency for both coated fabrics may be linked with the complete saturation of inherent nanopores after numerous cycles. This was possible since this coated fabric acted as a filter membrane with opposite wettability to aqueous phase while allowing for preferential absorption of the organic oil phase. ${ }^{\mathbf{4 , 3 0 , 3 1}}$ The observed degree of permeation of solvents through the nanopores of these coated fabrics in this test was due to differences in solvent weights. The observed trend could also be attributed to gravityassisted percolation as well as the contributions of superhydrophobic coated fabrics. ${ }^{4}$ Comparative barrier performances of some recent PDMS-based coated cotton fabrics within this study and in those reported the literature is presented in Table $3 .^{32-39}$

\section{Conclusions}

New superhydrophobic self-cleaning siloxane/ polydimethylsiloxane hybrid nanocomposite coatings were synthesized using two distinct silylating agents (HMDS and TMOS) to achieve enhanced superhydrophobicity when deposited on cotton fabrics. Variants of these coatings were also prepared by varying the amounts of both silylating coating components. After characterization and testing, the following conclusions were derived from the results of the experiments:

1. The hydrophilic cellulosic chemical groups on the uncoated cotton fabric contributed to its ability to readily adsorbed water. However, the coated silylated superhydrophobic coated fabrics demonstrated unique self-cleaning capacities due to their low surface energies and highly reduced surface wettability.

2. Coated cotton fabrics with higher TMOS contents (CMF14) demonstrated significant resistance against thermal oxidation due to their increased nanosilica composition. However, there was also reduced magnitudes of $\theta_{\mathrm{w}}^{\circ}$ at higher treatment 
temperatures. These coated fabrics were still hydrophobic $\left(\theta_{\mathrm{w}}^{\circ}>90^{\circ}\right)$ at the end of the tests. However, for CTF1-4 matrix (with higher HMDS content), the aqueous contact angle reduced remarkably after treatment to temperatures up to 40$70{ }^{\circ} \mathrm{C}$ due to pyrolysis of their organic contents.

3. Coated fabrics with higher TMOS content did not retain their superhydrophobicity $\left(\theta_{\mathrm{w}}^{\circ}=150^{\circ}\right)$ after acid and alkaline treatments. Both corrosive solvents had detrimental effects on both sets of coated fabrics within the duration of the test.

4. The observed superhydrophobic characters, solvent and thermal stabilities, capacities for self-cleaning and oil-water separation are ascribed to their nanopatterned nanosilica coated morphologies and low surface energy silylated hybrid polysiloxane chemical groups on these coatings.

5 . Coated superhydrophobic fabrics with $30 \mathrm{mg}$ TMOS $/ 10 \mathrm{mg}$ HMDS (CMF3) and $30 \mathrm{mg}$ HMDS/10 mg TMOS (CTF3) exhibited the optimal superhydrophobicity. Both fabrics also retained percentage separation efficiencies over 90\% for both chloroform-water and toluene-water filtered mixtures. However, CTF3 displayed less than $\left(\theta_{\mathrm{w}}^{\circ}<150^{\circ}\right)$ with recorded separation efficiency less than $90^{\circ}$ at the fifth consecutive separation cycle.

6. The substituted silyl $\left(\mathrm{Me}_{3} \mathrm{Si}\right)$ and octadecyl chemical groups on HMDS and TMOS contributed to enhanced superhydrophobicity. However, it was the hydrolysable trimethoxy silyl ((MeO) $\left.)_{3} \mathrm{Si}\right)$ chemical groups on TMOS that allowed for the formation of nanosilica with $\mathrm{Si}-\mathrm{O}-\mathrm{Si}$ linkages needed to foster stable coatings.

\section{Symbols, abbreviations and acronyms}

\begin{tabular}{|c|c|}
\hline$\gamma_{\mathrm{LA}}$ & Surface energy of liquid-air interface \\
\hline$\gamma_{\mathrm{SA}}$ & Surface energy of the surface-air interface \\
\hline$\gamma_{\mathrm{SL}}$ & Surface energy of surface-liquid interface \\
\hline$\theta_{\mathrm{o}}$ & Contact angle of water on the surface \\
\hline$R_{\mathrm{f}}$ & $\begin{array}{l}\text { Ratio of surface area of rough surface to the surface } \\
\text { area of a flat projection of the same surface }\end{array}$ \\
\hline$f_{\mathrm{LA}}$ & $\begin{array}{l}\text { Liquid-air fraction, the fraction of the liquid droplet } \\
\text { that is in contact with air }\end{array}$ \\
\hline$\theta_{\mathrm{CB}}$ & $\begin{array}{l}\text { Contact angle of water predicted by Cassie-Baxter's } \\
\text { model }\end{array}$ \\
\hline$\theta$ & Contact angle of water predicted by Wenzel's model \\
\hline AgNPs & Silver nanoparticles \\
\hline AgNWs & Silver nanowires \\
\hline EPMM & 3-(2,3-Epoxypropoxypropyl)methyldimethoxysilane \\
\hline EtPDMS & Ethoxy terminated polydimethylsiloxane \\
\hline FTIR & Fourier transform infrared \\
\hline HMDS & Hexamethyldisilazane \\
\hline MWCNTs & Multiwalled carbon nanotubes \\
\hline NPs & Nanoparticles \\
\hline $\mathrm{SiO}_{2} \mathrm{NPS}$ & Silicon oxide nanoparticles \\
\hline $\mathrm{TiO}_{2} \mathrm{NPs}$ & Titanium oxide nanoparticles \\
\hline TMCS & Chlorotrimethylsilane \\
\hline TMOS & Trimethoxy(octadecyl)silane \\
\hline UHV & Ultra-high vacuum \\
\hline XPS & X-ray photoelectron spectroscopy \\
\hline $\mathrm{ZnO}$ & Zinc oxide \\
\hline
\end{tabular}

\section{Conflicts of interest}

There are no conflicts of interest.

\section{Acknowledgements}

Authors wish to acknowledge University of Saskatchewan for providing some of the facilities for this study. This study was initiated and completed while UE was still at the Department of Mechanical Engineering, University of Saskatchewan, Canada.

\section{References}

1 P. Wang, J. Zhang, H. Wen, Z. Zhu, W. Huang and C. Liu, Colloids Surf., A, 2021, 610, 125684.

2 C. Cao, F. Wang and M. Lu, Colloids Surf., A, 2020, 601, 125033.

3 U. Eduok, O. Faye and J. Szpunar, Prog. Org. Coat., 2017, 111, 124-163.

4 A. Zhang, B. Liu, S. Liu, M. Li, W. Zheng, L. Deng and J. Liu, Chem. Eng. J., 2019, 371, 876-884.

5 N. Jannatun, A. Taraqqi-A-Kamal, R. Rehman, J. Kuker and S. K. Lahiri, Eur. Polym. J., 2020, 134, 109836.

6 S. S. Latthe, P. Sudhagar, A. Devadoss, A. M. Kumar, S. Liu, C. Terashima, K. Nakata and A. Fujishima, J. Mater. Chem., 2015, 3, 14263-14271.

7 H. Wang, M. He, H. Liu and Y. Guan, ACS Appl. Mater. Interfaces, 2019, 11, 25586-25594.

8 Y. Teng, Y. Wang, B. Shi, X. Li and Y. Chen, Polym. Test., 2020, 91, 106810.

9 X. Zhou, Z. Zhang, X. Xu, X. Men and X. Zhu, Appl. Surf. Sci., 2013, 276, 571-577.

10 S. Barthwal, S. Barthwal, B. Singh and N. B. Singh, Colloids Surf., A, 2020, 597, 124776.

11 H. Kang, B. Zhao, L. Li and J. Zhang, J. Colloid Interface Sci., 2019, 544, 257-265.

12 W. Bai, H. Lin, K. Chen, J. Xu, J. Chen, X. Zhang, R. Zeng, J. Lin and Y. Xu, Sep. Purif. Technol., 2020, 253, 117545.

13 P. Talebizadehsardari, J. Seyfi, I. Hejazi, A. Eyvaziand, M. Khodaie, S. Seifi, S. M. Davachi and H. Bahmanpour, Colloids Surf., A, 2020, 603, 125204.

14 W. Guo, X. Wang, J. Huang, Y. Zhou, W. Cai, J. Wang, L. Song and Y. Hu, Chem. Eng. J., 2020, 398, 125661; D. W. Wei, H. Wei, A. C. Gauthier, J. Song, Y. Jin and H. Xiao, J. Bioresour. Bioprod., 2020, 5, 1-15.

15 E. K. Sam, D. K. Sam, X. Lv, B. Liu, X. Xiao, S. Gong, W. Yu, J. Chen and J. Liu, Chem. Eng. J., 2019, 373, 531-546.

16 E. Pakdel, J. Wang, S. Kashi, L. Sun and X. Wang, Adv. Colloid Interface Sci., 2020, 277, 102116.

17 S. P. Dalawai, M. A. S. Aly, S. S. Latthe, R. Xing, R. S. Sutar, S. Nagappan, C. S. Ha, K. K. Sadasivuni and S. Liu, Prog. Org. Coat., 2020, 138, 105381.

18 S. S. Ugur, M. Sarısık and A. H. Aktas, Nanotechnology, 2020, 21, 325603, DOI: 10.1088/0957-4484/21/32/325603.

19 M. J. Juan-Díaz, M. Martínez-Ibánez, M. HernándezEscolano, L. Cabedo, R. Izquierdo, J. Suay, M. Gurruchaga and I. Goni, Prog. Org. Coat., 2014, 77, 1799-1806. 
20 M. S. Oliver, K. Y. Blohowiak and R. H. Dauskardt, J. Sol-Gel Sci. Technol., 2010, 55, 360-368.

21 I. R. Fontinha, M. M. Salta, M. L. Zheludkevich and M. G. S. Ferreira, Port. Electrochim. Acta, 2013, 31, 307-319.

22 A. V. Naumkin, A. Kraut-Vass, S. W. Gaarenstroom and C. J. Powell, NIST X-ray photoelectron spectroscopy database, NIST standard reference database 20, Version 2012, 2012, vol. 4.

23 Š. Meškinis, A. Vasiliauskas, M. Andrulevicius, D. Peckus, S. Tamulevicius and K. Viskontas, Materials, 2020, 13, 1-15. 24 J. Soethoudt, S. S. Crahaij, T. Conard and A. Delabie, J. Mater. Chem. C, 2019, 38, 11911-11918.

25 S. Meškinis, S. Tamulevicius, V. Kopustinskas, M. Andrulevicius, A. Guobiene, R. Gudaitis and I. Liutviniene, Thin Solid Films, 2007, 515, 7615-7618.

26 Y. Hou, Z. Wang, J. Guo, H. Shen, H. Zhang, N. Zhao, Y. Zhao, L. Chen, S. Liang, Y. Jin and J. Xu, J. Mater. Chem. A, 2015, 3, 23252-23260.

27 U. Eduok, R. Suleiman, M. Khaled and R. Akid, Prog. Org. Coat., 2016, 93, 97-108.

28 G. Guo, L. Liu, Q. Zhang, C. Pan and Q. Zou, New J. Chem., 2018, 42, 3819-3827.
29 A. K. Singh and J. K. Singh, New J. Chem., 2017, 41, 46184628.

30 Z. Shi, W. B. Zhang, F. Zhang, X. Liu, D. Wang, J. Jin and L. Jiang, Adv. Mater., 2013, 25, 2422-2427.

31 C. F. Wang and L. T. Chen, Langmuir, 2017, 33, 1969-1973. 32 S. Foorginezhad and M. M. Zerafat, J. Colloid Interface Sci., 2019, 540, 78-87.

33 X. Liao, H. Li, X. Lai, W. Chen and X. Zeng, Mater. Lett., 2019, 255, 126511.

34 Y. N. Gao, Y. Wang, T. N. Yue, Y. X. Weng and M. Wang, J. Colloid Interface Sci., 2021, 582, 112-123.

35 D. Lin, X. Zeng, H. Li, X. Lai and T. Wu, J. Colloid Interface Sci., 2019, 533, 198-206.

36 L. Zheng, X. Su, X. Lai, W. Chen, H. Li and X. Zeng, Mater. Lett., 2019, 253, 230-233.

37 T. Zhu, S. Li, J. Huang, M. Mihailiasa and Y. Lai, Mater. Des., 2017, 134, 342-351.

38 S. Fu, H. Zhou, H. Wang, J. Ding, S. Liu, Y. Zhao, H. Niu, G. C. Rutledged and T. Lin, RSC Adv., 2018, 8, 717-723.

39 A. K. Singh and J. K. Singh, $R S C$ Adv., 2016, 6, 103632103640. 